\title{
Monitoring malodours in the environment with an electronic nose: requirements for the signal processing.
}

\author{
Romain, A-C and Nicolas, $\mathrm{J}$ \\ Department of Environmental Sciences and management, Monitoring unit, \\ University of Liège, Avenue de Longwy 185, 6700 Arlon, Belgium, \\ acromain@ulg.ac.be
}

\begin{abstract}
:
Artificial olfaction system (the so-called electronic nose) is a very promising tool to monitor the malodour in the field. Usual measurement techniques of odour use human olfaction or conventional analytical techniques. The first category represents the real odour perception but is not applicable to measure continuously bad odours in the field. The second class of techniques gives the mixture composition but not the global information representative of the odour perception. The e-nose has the potentialities to combine "the odour perception" and the "monitoring in the field". However to be able to reach that goal, the signal processing has to be adapted to work in complex environment. The research group in Arlon has more than ten years experience in the measure of environmental malodours in the field. The paper presents the minimal requirements that the group considers as essential for artificial olfaction system to become successful for this application.
\end{abstract}

\section{Introduction}

Among all pollution problems, odour annoyance is considered as an important environmental issue since it induces a great number of complaints and conflicts between the residents and the industries. That growing environmental concern has led local authorities to consider odour policies to regulate the odour annoyance. Efforts to manage odour problems and to try to limit the exposure in the neighbourhood are necessary and, of course, the identification and the quantification of odour 
emission and exposure are very important aspects concerning the compliance with environmental regulation. As the sensitivity to odour involves the highly subjective reaction of individual persons, developing and testing reliable measurement techniques constitute really important challenges when dealing with olfactory pollution.

In this framework, the state-of-the-art generally reports two complementary types of measurement methods: human olfaction methods and conventional analytical techniques (Van Harreveld 2003, Lammers et al. 2004, Stuetz et al. 2001).

Human olfaction measurement considers the odour as a global concept and provides the true dimensions of the human perception. Yet, physiological differences in the smelling of various people often lead to subjective results with large uncertainties. Analytical techniques identify the various volatile compounds involved in the odour and give their chemical concentration. They have better scientific standing than sensory methods. However, the chemical composition of the gas mixture doesn't represent the odour perception.

A third and original concept is promising for measurement of malodour in the environment: the artificial olfaction. The so-called "electronic nose" instrument emerged at the beginning of the nineties thanks to some analogies with the biological sensing system. The instrument, based on non-specific gas chemical sensors array combined with a chemometric processing tool provides a suitable technique for in site monitoring of malodours. Published studies report attractive results (Bourgeois et al. 2003). However a number of limitations are associated with both the properties of chemical sensors and the performances of the signal processing. To meet the requirements of this environmental use of artificial olfaction system, the signal processing method must be simple, but not simplistic, and capable of generalisation. It must be tolerant to hardware weaknesses and adapted for application in the real life. 


\section{Usual methods to measure the malodour pollution}

\subsection{Human olfaction measurement}

\subsubsection{Odour dimensions}

Besides the stimulus, which is a mixture of volatile compounds at given concentrations, the processing of the odour information by the brain is rather complex and leads to a multidimensional sensation. Three main dimensions can be considered: the intensity, or the "strength" of the odour, the quality, or the nature of the odour and the hedonic tone, or the affective reaction to the odour.

Intensity refers to the perceived strength of the odour sensation. To generate such perception, the physical stimulus, i.e. the mixture of odorous molecules, must be detectable. That means that its concentration must exceed a given threshold.

The relationship between the perceived intensity and the concentration of the stimulus, or the odour concentration, is non-linear and depends on the nature of the odorants.

Two famous psychophysical laws express this relationship: the Stevens and the Weber-Fechner laws (Nicolas 2001, Misselbrook et al. 1993, Sperber et al. 2003).

The intensity can be measured by ranking the odour impression on a predetermined scale or by comparison with a series of samples of known concentration of a reference substance (see for example VDI guideline 3882 -1997- Determination of odour intensity).

The notion of odour concentration is based on the works of $\mathrm{H}$. Zwaardenmaker, a Dutch scientist and early investigator of the olfactometry. By definition, the odour concentration, expressed in odorous unit per cubic meter $(\mathrm{ou} / \mathrm{m} 3)$ is the dilution factor of the odour sample in clean air in order to just become odour free, i.e. to reach the perception threshold for an "average" person.

The dynamic olfactometry is the official method by which different dilutions of the gas sample are dynamically presented to trained assessors to determine the odour concentration of the original sample. When the European standard method (EN13725 -European standard "air quality"Determination of odour concentration by dynamic olfactometry, 2003) is used, the concentration is expressed in $\mathrm{ou}_{\mathrm{E}} / \mathrm{m} 3$ (with the subscript E). 
The second dimension of the odour is its quality, expressed in descriptors, or words that describe the smell, such as fruity, woody, sour, pungent (Dravnieks 1992). Contrary to perfumery or oenology, there isn't any standardised list of words to qualify environmental odours and the odour quality is usually associated with its origin, i.e. the emission source.

The third dimension is the hedonic tone, or the category of judgement of the relative like (pleasantness) or unlike (unpleasantness) of the odour. That is an emotional level of reaction which is assessed in accordance with a given category scale, from "extremely unpleasant" to "extremely pleasant" (see for example VDI guideline 3882-2-1994- Determination of hedonic odour tone).

\subsubsection{Odour annoyance criteria}

More specifically for environmental odours, the whole process from the formation of odorants to the complaint action may be complex. It implies many contributing factors and conditions at the different steps.

So, the terminology usually distinguishes the annoyance from the nuisance. Annoyance is simply the negative appraisal of an odour, but nuisance is the cumulative effect on humans, caused by repeated events of annoyance over an extended period of time, that leads to modified or altered behaviour. And, finally, significant odour nuisance may trigger a complaint to a regulatory authority.

Five factors are generally identified in relation to odour impact (Hayes et al. 2006, Schauberger et al. 2001).

Those are the five "FIDOR" (or "FIDOL") factors:

-Frequency, or the number of times an odour is detected over a specific time period,

-Intensity, or the strength of the odour, as defined above,

-Duration, or the length of exposure,

-Offensiveness, or the hedonic tone,

-Receptor, (or "Location") including the physiological, social and economical aspects of the individual perceiving the odour.

Besides the quantitative and qualitative dimensions of the odour, the annoyance is thus chiefly governed by the tolerance and the expectation of the receptor as well as by the time dimension. 
The location of the odour event receptor determines how objectionable the odour event is. In some locations certain odours may be more acceptable than in others. For example, breeding smells may be better accepted in rural area or a given industrial smell sometimes does not generate complaints if the company is a job provider for the region.

Concerning the time dimension, frequency and duration may be measured using odour-hours, i.e. the number of hours of odour perception for a given location.

Time variation is actually one of the main specificities of environmental odours, which could invalidate values obtained only by some spot measurements.

For instance, some investigations carried out on 9 landfill sites in Belgium showed that a wide variety of odour emissions are generated by waste. They are conveniently separated into the specific activities, such as active tipping of the waste, its transportation by disposal trucks, its intermediate storage and the handling process after the garbage deposit. On the investigated sites the main odour problem was caused by the handling of the fresh waste, which is an intermittent activity and which makes difficult the sampling of the gas emitted at the landfill working face (Nicolas et al. 2006).

\subsection{Conventional Analytical techniques}

Chemical analysis like gas chromatography coupled to mass spectrometry (GC-MS) carried out in the lab on the basis of samples collected on adsorbent cartridges does not allow monitoring the odour fluctuation in real time.

Alternatively, hand-held specific field detectors allow direct measurement and continuous operation. Particular volatiles, such as hydrogen sulphide, ammonia or total reduced sulphur (TRS), can be continuously recorded and a sudden signal rise can be considered as a sign of the odour emergence for a given source.

However, it is true only if the particular volatiles are correlated with the concentration of the odour of interest. Measuring odours with too specific gas detectors is only suited for emissions with well known gaseous compositions. Moreover, for industrial sites characterised by various gas releases, different types of emission can generate the same signal. 


\section{Artificial olfaction for measurement of odour pollution}

\subsection{Interest for environmental application}

Artificial, but biological-inspired odour measurement methods constitute attractive alternatives to the use of human panels to assess the malodour in the environment. The evaluation of odour annoyance in the field requires approaches more objective.

The electronic nose principle exploits that lack of relative specificity to typical gaseous analytes by considering the pattern of sensor signals as global response to the gas mixture. Such bioinspired strategy opened the door in the early eighties to new prospects for those devices that try to mimic the human olfactory system (Persaud 1982, Gardner 1991). They include indeed similar corresponding components: the array of chemical sensors, the data processing unit and the pattern recognition engine respectively for the olfactory receptor cells, the olfactory bulb and the brain. Many pattern recognition techniques used to identify the signal patterns apply also bioinspired algorithms, such as artificial neural networks.

One of the main advantages of the electronic nose for malodour measurement is the possibility to use it as a field continuous monitor of odorous emissions (Nicolas and Romain 2000). This technique has probably the best potentialities to answer to the expectations of the various actors of the environmental problems in relation with the odour annoyance.

\subsection{Stepwise methodology}

To develop an artificial olfaction system and to build the pattern recognition and the odour regression model for this kind of application, we use a stepwise methodology

The first action to be undertaken is a visit in the field in order to investigate the problem. Then the selection come and the development of the method according to the field criteria and limits. First options are taken and a first prototype is developed and tested in the lab.

A second field campaign is then organised to test the system and to gather some preliminary data to get some proofs before going back a second time in the lab and improving and correcting eventual identified problems. 
When the instrument is considered good enough to be carried in the field, then, the learning phase begins. It requires the presence of the operator in the field during several hours and several days to validate each sensor signal variation by sensory observations. Such stage is fundamental to feed the pattern recognition procedures with sufficient data, based on a huge number of various ambiences, to build robust classification and regression models.

A further step in the lab aims at verifying the models in different conditions and with various observation samples. Then a last validation step is carried out in the field.

Such to-and-fros between field and lab results in a final system able to meet field requirements and to bring an optimum solution to the problem. That quite heavy process must be repeated for each new application, because e-nose is not a universal tool. The only way to guarantee accurate recognition and/or odour concentration prediction is to dedicate the instrument to a specific utilisation. Hardware and signal processing dedicated to a precise application are the keys of the e-nose credibility.

\subsection{Variability of operating conditions}

Real life field conditions are never reproducible. For the same site of interest, diverse activities, different process management strategies or various raw material compositions induce highly variable emissions. As a result, for one odour source, there are many different mixtures of compounds.

So, the various observations belonging to one class of signal patterns are far from being homogeneous.

Figure 1 shows two different chromatographic profiles for the same malodorous source. The two samples were collected from the ambience of a shelter of a household waste composting plant. The sampling was realised in the same location but during two different days.

VOC's are sampled on Tenax ${ }^{\mathrm{TM}}$ tubes during 20 min with a flow of 200 $\mathrm{ml} / \mathrm{min}$ and then desorbed using a thermal desorber (UNITY ${ }^{\mathrm{TM}}$ from Markes International) linked to a gas chromatograph coupled to mass spectrometer (Agilent HP6890 GC and 5972 MS). The carrier gas is helium. The column CP-Sil 8 CB MS used (5\% phenyl and 95\% dimethylpolysiloxane) (Chrompack) has a length of $50 \mathrm{~m}$, internal diameter $0.25 \mathrm{~mm}$, and film thickness of $0.4 \mu \mathrm{m}$. Running conditions for GC are: initial temperature $38{ }^{\circ} \mathrm{C}$; initial time $7 \mathrm{~min}$; rate $5{ }^{\circ} \mathrm{C} / \mathrm{min}$; final 
temperature $250{ }^{\circ} \mathrm{C}$; and final time $5 \mathrm{~min}$. The MS transfer line is at $280^{\circ} \mathrm{C}$ and the mass scan range is from 29 to $300 \mathrm{amu}$

About 200 compounds are identified in each sample. They correspond to 15 chemical classes.

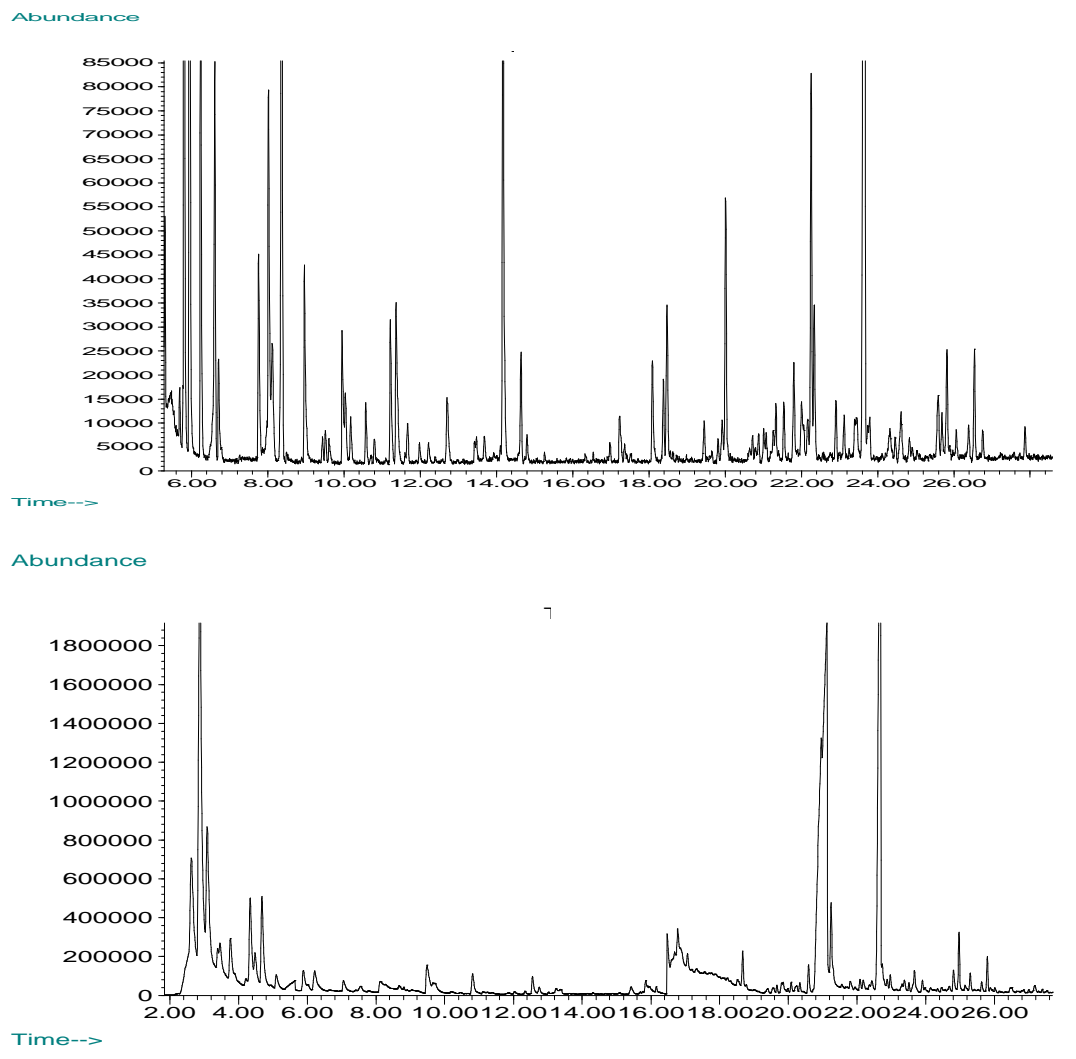

Figure 1. Gas chromatograms obtained by sampling the same odorous source (in a compost shelter) two different days.

This figure demonstrates the diversity of the chemical composition for one malodour source.

Other sources of variability are the meteorological conditions. They influence the rate and the composition of the generated odour and affect its dispersion. But they also exert an effect on the gas sensor behaviour (e.g.: signal variation due to humidity change). 
Each of those temporal disturbances induces specific signal variation. It contains relevant pieces of information, of different types, and which should not be confused with noise, which is random and erratic. So, to be able to recognise the malodour in the background, among other gaseous emissions, it is essential to extract just the signal pattern corresponding to the targeted emission. Also it is fundamental to appraise a significant deviation of the global response from the "normal" margin.

Various actions are considered by the identification of the specific variations mentioned above: sending a warning signal when the odour level rises up above a worrying threshold, assessing an annoyance zone in the surroundings on the basis of the sensor responses or using the measured signals to control an odour abatement technique

Figure 2 illustrates the time evolution of the conductance of tin oxide gas sensors placed in the ambience of a composting process.

The self-made electronic nose consists in a sensor array and a PC board, with a small keyboard and a display. Six commercial metal oxide sensors (Figaro ${ }^{\circledR}$ ) are placed in a rectangular $160 \mathrm{~cm}^{3}$ stainless steel chamber. The sensors were selected on the basis of some operating criteria among the range of sensors proposed by the Japanese manufacturer Figaro and chiefly among the $12 \mathrm{SnO}_{2}$ sensors used in previous studies (Romain 2000). Some sensors were eliminated for their too low sensitivity towards compost emissions: relative R/R0 resistance variations near zero caused the removal of two sensors. Two ones were eliminated because of there too long recovery time, their poor stability or their too large signal to noise ratio and an additional one for its redundancy with TGS 842. Finally the selected sensors were those for which the contribution to the discrimination power between compost and background air was the highest. When two sensors were available for the same purpose, one from the " 800 " series and the other one from the " 2000 " series, this last one was preferred for its low electrical consumption. The six selected sensors are listed in Table 1.

Though TGS 2180's response to compost emission was low, this sensor was kept if a signal for humidity correction was needed.

The chamber temperature is kept at $60 \mathrm{C}$ by a heating resistor and natural cooling, thanks to a suitable control system. Relative humidity of the sensor chamber is also recorded. The ambient air is sucked in though a teflon tubing with a flowrate of $200 \mathrm{ml} / \mathrm{min}$ thanks to a small pump controlled by the computer code. Data are recorded in the local memory and downloaded in an external computer to be off-line processed by statistical and mathematical tools (Statistica and Matlab). 


\begin{tabular}{ll}
\hline Sensor & Applications (from the manufacturer) \\
\hline TGS822 & Organic solvents (ethanol, benzene, acetone, ...) \\
TGS880 & Volatiles vapour from food (alcohols) \\
TGS842 & Natural gas, methane \\
TGS2610 & Propane, butane \\
TGS2620 & Hydrogen, alcohols, organic solvents, \\
TGS2180 & Water \\
\hline
\end{tabular}

Table 1 the six selected Figaro sensors

Short time signal variations are clearly identified over the noise level and are due to local handlings of compost material. Some other external causes induce diurnal or seasonal variation.

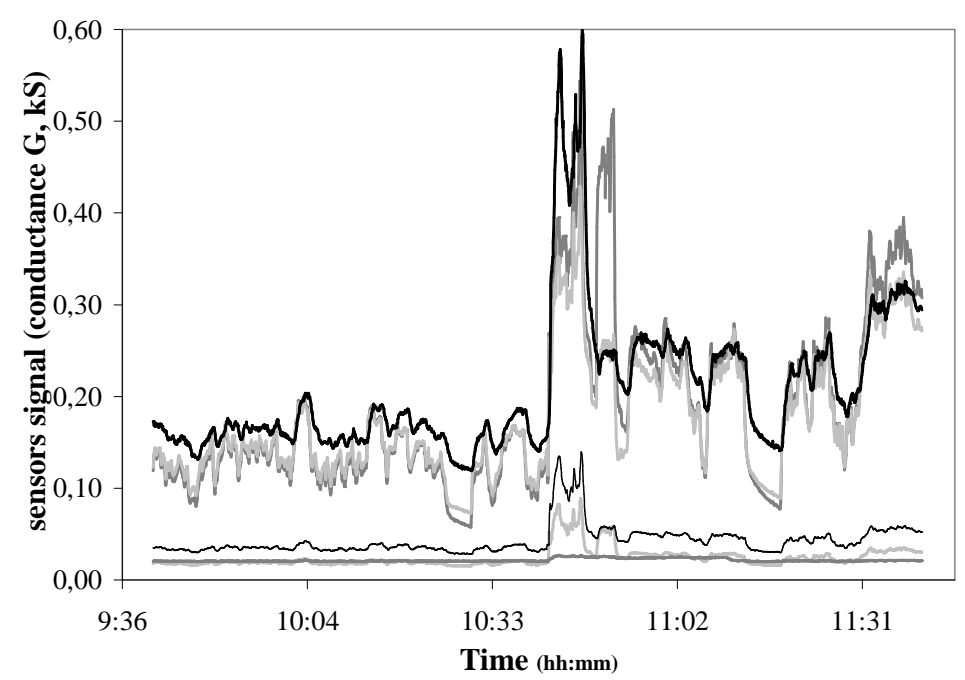

Figure 2. Example of time evolution of 6 tin oxide gas sensors (FigaroTM sensors) in the ambience of a composting plant.

For all those above reasons, trying to classify environmental odour sources in real life with pattern recognition techniques gives rise to a spread of observation points in the different clusters. For the signal processing, that implies to have a great number of samples in order to consider the various conditions and chemical compositions. But that induces a high dispersion of the data of a same class and/or of the same concentration.

The data dispersion is shown on principal component analysis (PCA) scores plot (figure 3 ). The projection in the two first components plane of 
the PCA highlights the effect of the variability of the operating conditions on the data.

Such dispersion is the normal image of the large number of samples, collected in many different conditions and with various chemical compositions.

In the figure, the points in the transitional region between "compost odour" and "odourless background" simply represent less odorous compost ambience or slightly loaded background ambiance.

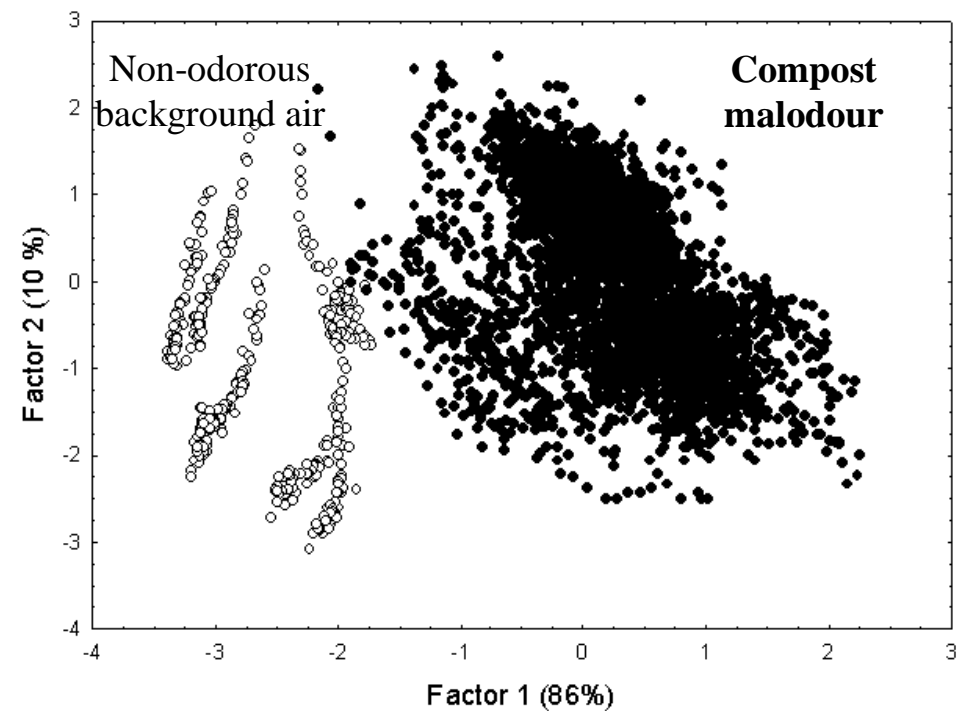

Figure 3. PCA sores plot for measurements with a lab-made e-nose (array of 6 Figaro $^{\mathrm{TM}}$ sensors) in a composting plant. The high variance of the data is due to the variability of the operating conditions.

Moreover, to be able to operate in the environment as an efficient standalone instrument, with minimum maintenance, the hardware of the electronic nose has to cope with harsh ambient conditions: dust, humidity, vibration, etc.

And, last but not least, one of the most severe requirements for an instrument aiming at detecting olfactory annoyance is the fact that contrary to many chemical applications- the final expected useful information is the odour dimension of the gas emission, and not the concentration of some chemicals. 


\section{Requirements for the signal processing when using artificial olfaction instrument in the environment}

\subsection{Context}

As a result of harsh environmental conditions, of hardware limitations and of olfactory pollution specificities, odour real-time monitoring with an electronic nose is thus a real challenge.

The instrument has to cope with several specific drawbacks. It has to automatically compensate the time drift and the influence of ambient parameters, such as temperature or humidity, to filter the unwanted signal variation due to the normal evolution of the background chemical composition, to experiment a huge number of various ambiences during the learning phase, to extract just the signal pattern corresponding to the targeted emission, to appraise a significant deviation of the global response from the "normal" margin and to supply final indicators which aggregate the response of the sensor array into a global odour index.

Although some of those problems may be tackled through an hardware approach (improvement of sensor performances, optimisation of sensor chamber, control of gas line, etc.), most of them must be solved by a suitable on-line signal processing directly embedded in the field instrument.

\subsection{Sensor drift and calibration gas}

\subsubsection{Drift}

Sensor drift is a first serious impairment of chemical sensors. They alter over time and so have poor repeatability since they produce different responses for the same odour. That is particularly troublesome for electronic noses (Romain et al. 2002). The sensor signals can drift during the learning phase (Holmberg et al. 1997). To try to compensate the sensor drift, three types of solutions were tested for our applications.

The usual way of minimising drift effect is to consider as useful response the difference between the base line, obtained by presenting the sensor array to pure reference air, and the signal obtained after stabilisation in the polluted atmosphere. However, such solution requires operating by cycling between reference air and tainted air, which is not convenient for on-site 
applications. That requires carrying in the field heavy gas cylinders.

Alternatively, generating the reference air by a simple filtering of ambient atmosphere gives rise to only partial drift compensation and to a lack of purity of the reference gas, which increase the data dispersion.

Posterior global drift counteraction algorithms could be applied either for each individual sensor or by correcting the whole pattern, using multivariate methods.

First the main direction of the drift is determined in the first component space of the multivariate method, such as Principal Component Analysis (PCA), or by selecting time as dependant variable of a Partial Least Square regression (PLS). The drift component can then be removed from the sample gas data, correcting thus the final score plot of the multivariate method (Artursson et al. 2000).

Univariate sensor correction gave the best results in our case (table 2). With real-life measurements, it is indeed very difficult to identify a single direction in a multivariate space that is only correlated to sensor drift. So, for each sensor, an individual multiplicative factor was calculated by estimating the drift slope for a standard gas.

\subsubsection{Calibration gas}

However, an additional difficulty arises when having to select the adequate calibration gas. There is actually no standard for "compost odour" or "printing house odour".

Criteria to choose a reliable calibration gas are: as simple mixture as possible, with well known compounds, easy to obtain and not too expensive, reproducible, time stable, and, of course, which generates sensor responses similar to the ones obtained with the studied odour. As it should be difficult to produce stable artificial gas mixture, the best way is to select a single gas. 
Figure 4 shows drift compensation of a commercial tin oxide sensor (TGS2620, Figaro ${ }^{\mathrm{TM}}$ ) by multiplicative factor estimated from calibration measurements in ethanol.

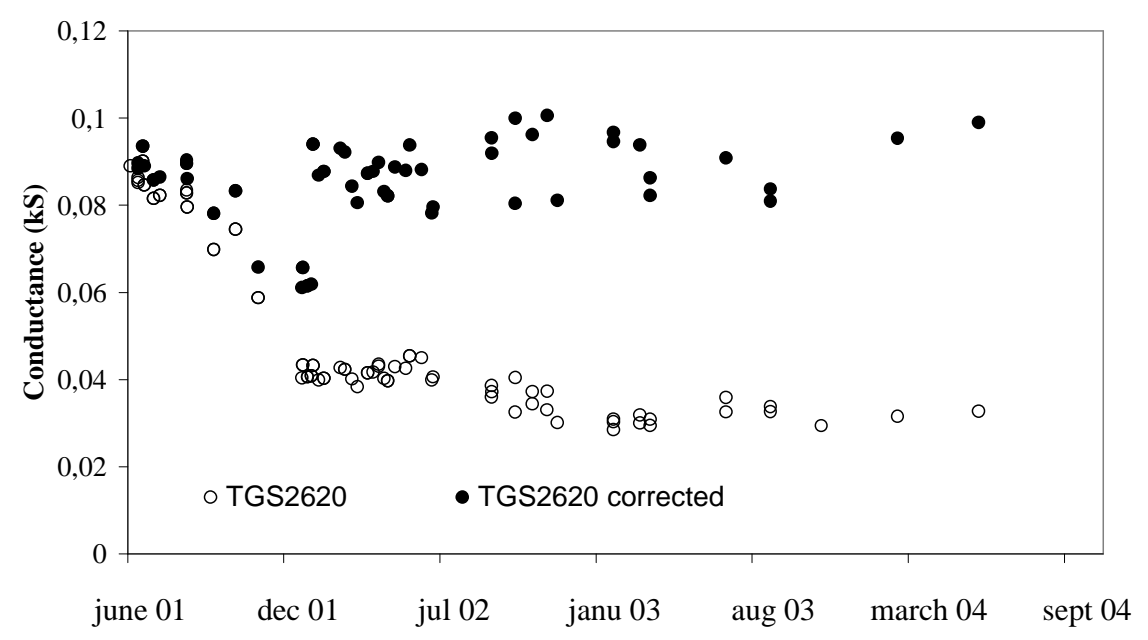

Figure 4. Drift correction of the sensor TGS 2620 by multiplicative factor estimated by ethanol measurements.

The multiplicative factor looks to be able to compensate the drift of this sensor for measurement in ethanol.

In order to test the performance of the method with this calibration gas on malodour measurements, we apply linear discriminant analysis (LDA or fisher discriminant analysis) for two different environmental odours: a compost odour and a printing house odour. Measurements are obtained by an array of 5 tin oxide sensors.

Linear discriminant analysis (LDA) was used with 5 features ( 5 sensor conductance values) and 63 observations collected within a 22- month's period. Two classes were considered (compost class and printshop class) The Fisher-Snedecor F-ratio of intergroup/intragroup variances was chosen as classification performance criterion. Higher the F value is, better is the separation of the classes. 
Table 2 illustrates the results obtained for the three types of solutions presented in the paragraph 4.2.1 above.

\begin{tabular}{l|c}
\hline Method & F \\
\hline No correction & 33 \\
\hline Correction by sensor (individual multiplicative factor) & 56 \\
\hline Correction of the sensor array "PLS" & 26 \\
\hline Correction of the sensor array "PCA" & 18 \\
\hline
\end{tabular}

Table 2 Evaluation of the classification without correction or with correction models (by the $\mathrm{F}$ criterion, F-ratio of intergroup/intragroup variances).

The greatest value of $F(56)$ and consequently the best separation between the printshop data set and the compost one is obtained after the correction of the drift by individual multiplicative factor.

The worst performance is obtained after the "PCA sensor array" correction. The result is worse (18) with the correction than without drift compensation (33).

\subsubsection{Sensor replacement}

Another frequent problem encountered in the field and particularly in highly polluted atmosphere is a sensor failure or an irreversible sensor poisoning. Clearly, life expectancy of sensors is reduced for real-life operation with respect to clean lab operation. Sensor replacement is generally required to address such issue, but, after replacement, odours should still be recognised without having to recalibrate the whole system. But commercial sensors are rarely reproducible.

Figure 5 shows a PCA plot in the plane of the two first components. It concerns 260 observations, 3 classes (ethanol, background air and compost odour), 5 features and a 2-year period. After the replacement of the sensors in the array with the same trade mark references, the previous calibrated model is no longer applicable for the same odorous emissions: all the observation points are shifted to another part of the diagram. 


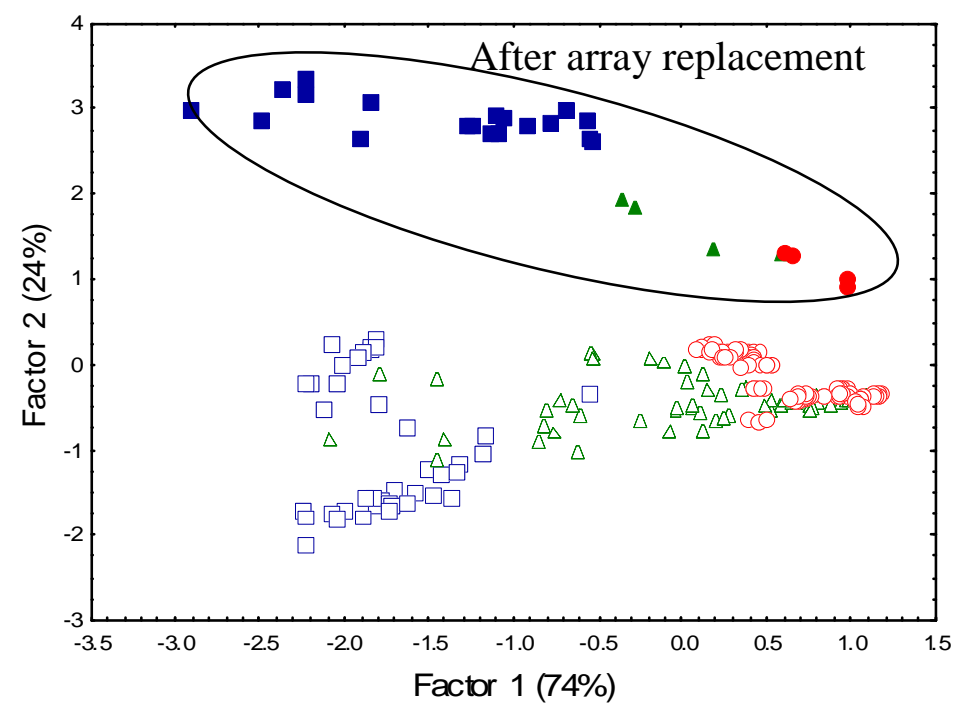

Figure 5. Illustration, by a PCA score plot of the shift of the measurements after the replacement of the TGS sensors

Again, correction routines including algorithms for handling shift related to sensor replacement can be successfully applied. For the above example, illustrated in figure 5, the classification performances were severely reduced after array replacement. The percentages of correct classification were $40 \%, 100 \%$ and $33 \%$ respectively for ethanol vapour, background air and compost emission. After individual sensor correction (with the same procedure individual multiplicative sensor than for drift correction), each classification rate reaches $100 \%$ (table 3 ).

\begin{tabular}{l|lc}
\hline Source & \multicolumn{2}{|c}{$\%{ }^{\text {age }}$ correct classification } \\
& \multicolumn{2}{|c}{ Validation data } \\
& without correct. & with correct. \\
\hline ethanol & 40 & 100 \\
\hline air & 100 & 100 \\
\hline compost & 33 & 100 \\
\hline
\end{tabular}

Table 3. Percentage of correct classification after replacement of sensors with and without correction. 


\subsection{Odour quantification}

One of the goals of odour monitoring with sensor arrays is the detection of odour emergence in the background. To be able to define some warning threshold or to assess potential annoyance zones in the surroundings, it is essential to establish a relationship between the e-nose response and the sensory assessment of the odour. But, again, such relationship cannot be unique for a given odour emission: one odorous source corresponds of many different chemical mixtures with variable composition. So, the " $x "$ variable of the relationship, i.e. the e-nose response to volatile compounds, is spread over a large range of possible values. But the "y" branch of the relationship, i.e. the odour sensory assessment, such as the odour concentration measured by dynamic olfactometry, is also affected by large uncertainties. Odour concentration is indeed the result of a rather subjective assessment with a panel of individuals.

Moreover, the gas sensors respond to odorous and odourless compounds. The condition for the global e-nose signal to be proportional to the "odour" is that the "chemical" concentration and the odour concentration are correlated. That is true for compost emission: there is a quite linear relationship between odour concentration and the response of the TGS 822 sensor (figure 6).

Odour concentration $\left(\mathrm{ou}_{\mathrm{E}} / \mathrm{m}^{3}\right)$

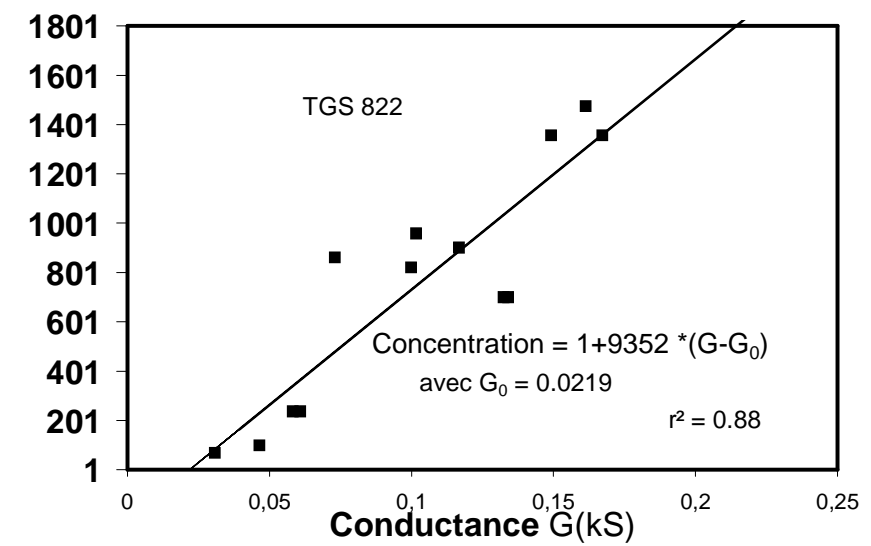

Figure 6. Regression model obtained for compost odour concentration

But that is not verified for the odour generated by a printing house (figure 7) because there are a lot of chemical compounds that are not odorous in the ambience. 
Odour concentration $\left(\mathrm{ou}_{\mathrm{E}} / \mathrm{m}^{3}\right)$

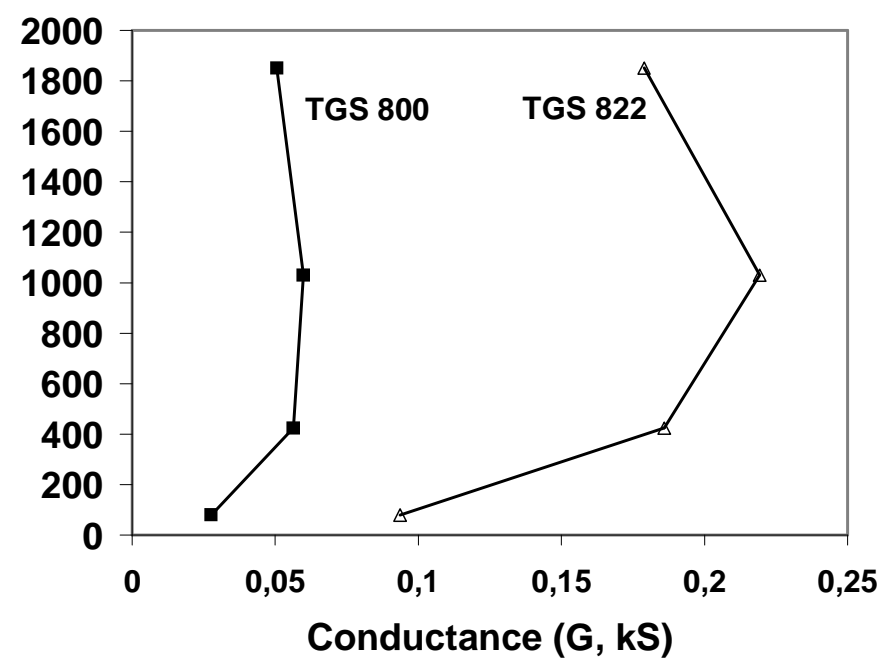

Figure 7. No regression model for a printshop odour

So, in the case of printing house, we cannot use the electronic nose to monitor the odour. But, in the case of compost emission, such monitoring should be possible as long as the relationship between odour and sensor response is kept after eventual sample preparation (eventual preconcentration, filtering, drying, etc.).

Figure 8 shows the evolution of the response of an electronic nose placed in a composting hall. Thanks to a suitable calibration model established with parallel olfactometric measurements, the global response is translated into odour concentration unit $\left(\mathrm{ou}_{\mathrm{E}} / \mathrm{m}^{3}\right)$ and could be compared to a warning threshold concentration, e.g. $700 \mathrm{ou}_{\mathrm{E}} / \mathrm{m}^{3}$. 


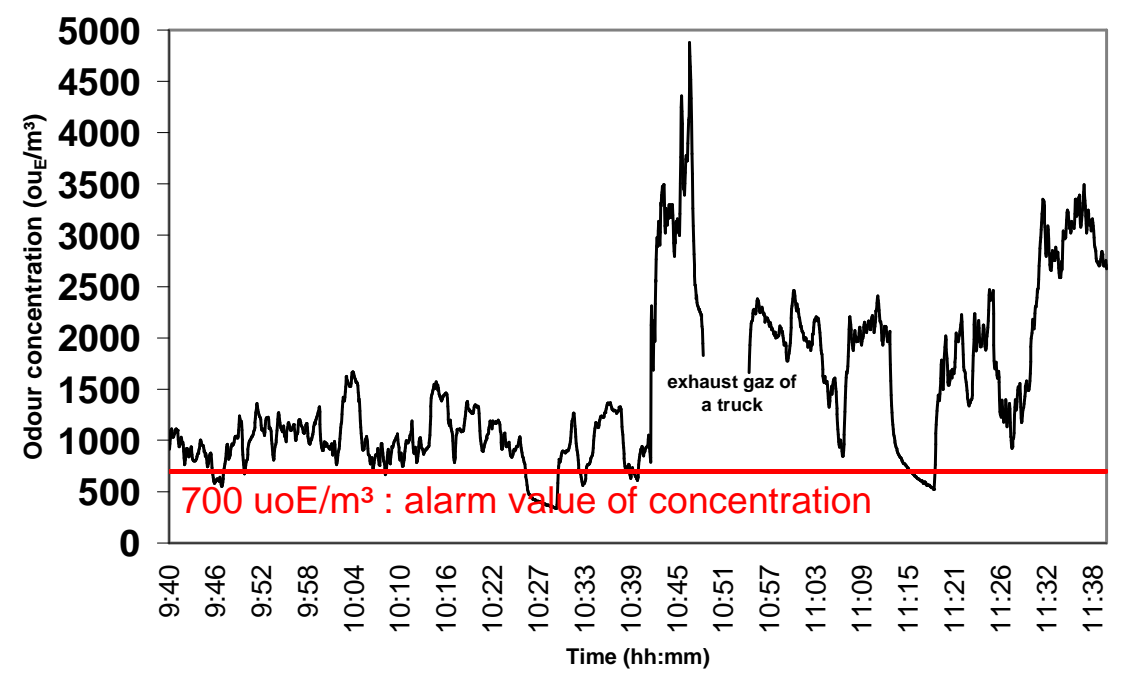

Figure 8. Monitoring of the compost odour concentration

\subsection{Useful signal detection}

A last typical example of specific problem when monitoring real-life odours with e-nose is the on-line detection of an abnormal signal emergence.

That issue could be addressed through correct filtering.

Figure 9 illustrates such signal processing for an array of sensors placed in the vicinity of settling ponds of a sugar factory. The aim of the filtering process is to keep only significant high frequency variations. This can be achieved simply by removing the low frequency part from the true signal. A first-order low pass filter is applied. For this filter, a time constant of about 150 seconds is applied. It is relevant for our system and our operating conditions. As such variations can be generated by many different causes; the real odour problem must be correctly identified. Combining the filtering method with a specific expert system allows such useful signal detection. 


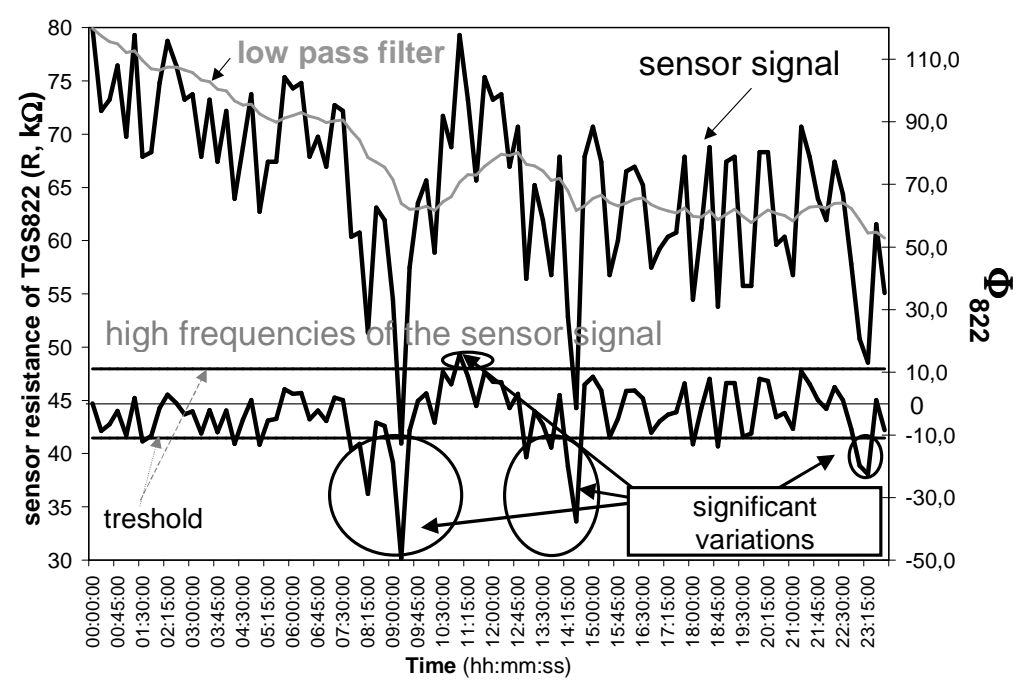

Figure 9. Thank to the filtration of the signal $(\Phi 822), 4$ events are detected (significant variations coupled to an expert system -not shown here-)

\section{Conclusion}

On-line use of electronic nose in the field aiming at monitoring real-life malodours is feasible, but has to correct many problems due to harsh environment and to the specificities of the olfactory annoyance assessment. Hopefully, the requirements of the final users seldom are as restricting as laboratory applications ones. Different signal processing techniques and many data classification methods were tested, but sufficiently good results are generally obtained with classical and simple techniques. Ideal methods commonly recommended by signal and data processing theories often are well suited for "clean data sets", with observations gathered in the rigorous conditions of the lab, but are rarely applicable with real-life environmental conditions.

The special nature of the environmental malodour monitoring always has to be considered when designing an instrument for such purpose: time variation of the emission, large number of odour categories, sensory dimension of the final variable to assess, lack of standard, etc.

Bio-inspired signal processing development is still necessary, but must be driven by some specific constraints: preliminary field investigation and subsequent on-line validation, even if it is time consuming and not easy, and ability to function in continuous in the field. 


\section{References}

Artursson, Tom, Eklov, Tomas, Lundström, Ingemar, Martersson, Per, Sjöström, M., Holmberg, M. (2000) Drift correction for gas sensors using multivariate methods. Journal of chemometrics, 14 711-723.

Bourgeois, W, Romain, A-C., Nicolas, J., Stuetz, R.M. (2003) The use of sensor arrays for environmental monitoring: interests and limitations. J. Environ. Monit., 5 6:852-860.

Dravnieks, Andrew., Atlas Of Odor Character Profiles.DS 61, American Society for Testing and Materials (ASTM), Philadelphia, 1992.

Gardner, J. W., Bartlett, P. N., Sensors and sensory systems for an electronic nose, 212, Kluwer Academic, London, 1991.

Hayes, E.T., Curran, T.P., Dodd, V.A. (2006) A dispersion modelling approach to determine the odour impact of intensive poultry production units in Ireland. Bioresource Technology, 97 15:17731779.

Holmberg, M., Davide, F. A. M., Di Natale, C., D'amico, A., Winquist, F., Lundstrom, I. (1997) Drift counteraction in odour recognition applications: Lifelong calibration method. Sensors and Actuators B: Chemical, 42 3:185-194.

Misselbrook, T.H., Clarkson, C.R., Pain, B.F. (1993) Relatioship between concentration and intensity of odours for pig slurry and broiler houses. J. Agric. Engng Res., 55 163-169.

Nicolas, J., Craffe, F., Romain, A.C. (2006) Estimation of odor emission rate from landfill areas using the sniffing team method. Waste Management, 26 11:1259-1269.

Nicolas, J., Romain, A.C. (2000) Using the classification model of an electronic nose to assign unknown malodours to environmental sources and to monitor them continuously. Sensors and Actuators B: Chemical, 69: 366-371.

Nicolas, S (2001) Gustav Theodor Fechner (1801-1887) et les précurseurs français de la psychophysique : Pierre Bouguer (1729) er Charles Delezenne(1828). Psychologie et histoire, 2 86-130.

Persaud, K. C., Dodd, G., Analysis of discrimination mechanisms in the mammalian olfactory system using a model nose, Nature, 299, (1982) 352-355

Romain, A.-C., Nicolas, J., Wiertz, V., Maternova, J., Andre, P., Use of a simple tin oxide sensor array to identify five malodours collected in the field, Sens. actuators, B, Chem., 62, 1 (2000) 73-79. 
Romain, A-C., André, Ph., Nicolas, J. (2002) Three years experiment with the same tin oxide sensor arrays for the identification of malodorous sources in the environment. Sensors and Actuators B: Chemical, 84:271-277.

Schauberger, G, Piringer, M, Petz, E (2001) Separation distance to avoid odour nuisance due to livestock calculated by the Austrian odour dispersion model (AODM). Agriculture, Ecosystems and environment, 87 13-28.

Sperber, G., Course : General sensory physiology, http://www.neuro.uu.se/fysiologi/gu/nbb/lectures/index.html, Uppsala University (Sweden), Department of Physiology, 2003, $11 / 03$

Stuetz, R. M., Frechen, F-B, Odours in wastewater treatment: Measuring, Modelling and control, IWA, London, 2001.

Van Harreveld, A. Ph. (Ton) (2003) Odor regulation and the history of odor measurement in Europe. In: (Ed.: N. a. V. Office of Odor, Environmental Management Bureau, Ministry of the Environment, Government of Japan), State of the art of odour measurement, pp. 54-61

Yuwono, Lammers (2004) Odor Pollution in the Environment and the Detection Instrumentation. the CIGR Journal of Scientific Research and development, 6. 